\title{
Clinical evaluation of Synthetic Aperture Sequential Beamforming and Tissue Harmonic Imaging
}

Brandt, Andreas Hjelm; Hemmsen, Martin Christian; Hansen, Peter Møller; Lindskov Hansen, Kristoffer; Lange, Theis; Jensen, Jørgen Arendt; Nielsen, Michael Bachmann

\section{Published in:}

Proceedings of IEEE International Ultrasonics Symposium

Link to article, DOI:

10.1109/ULTSYM.2014.0324

Publication date:

2014

Document Version

Early version, also known as pre-print

Link back to DTU Orbit

Citation $(A P A)$ :

Brandt, A. H., Hemmsen, M. C., Hansen, P. M., Lindskov Hansen, K., Lange, T., Jensen, J. A., \& Nielsen, M. B. (2014). Clinical evaluation of Synthetic Aperture Sequential Beamforming and Tissue Harmonic Imaging. In Proceedings of IEEE International Ultrasonics Symposium (pp. 1312 - 1315). IEEE.

https://doi.org/10.1109/ULTSYM.2014.0324

\section{General rights}

Copyright and moral rights for the publications made accessible in the public portal are retained by the authors and/or other copyright owners and it is a condition of accessing publications that users recognise and abide by the legal requirements associated with these rights.

- Users may download and print one copy of any publication from the public portal for the purpose of private study or research.

- You may not further distribute the material or use it for any profit-making activity or commercial gain

- You may freely distribute the URL identifying the publication in the public portal 


\title{
Clinical evaluation of Synthetic Aperture Sequential Beamforming and Tissue Harmonic Imaging
}

\author{
Andreas Hjelm Brandt ${ }^{\mathrm{a}, \mathrm{b}}$, Martin Christian Hemmsen ${ }^{\mathrm{b}}$, Peter Møller Hansen ${ }^{\mathrm{a}}$, Kristoffer Lindskov Hansen ${ }^{\mathrm{a}}$, \\ Theis Lange $^{\mathrm{c}}$, Jørgen Arendt Jensen ${ }^{\mathrm{b}}$, and Michael Bachmann Nielsen ${ }^{\mathrm{a}}$ \\ ${ }^{a}$ Dept. of Radiology, Copenhagen University Hospital, Denmark \\ ${ }^{\mathrm{b}}$ Center for Fast Ultrasound Imaging, Technical University of Denmark, Denmark \\ ${ }^{\mathrm{c}}$ Section of Biostatistics, University of Copenhagen, Denmark
}

\begin{abstract}
This study determines if the data reduction achieved by the combination Synthetic Aperture Sequential Beamforming (SASB) and Tissue Harmonic Imaging (THI) affects image quality. SASB-THI was evaluated against the combination of Dynamic Received Focusing and Tissue Harmonic Imaging (DRF-THI). A BK medical UltraView 800 ultrasound scanner equipped with a research interface and an abdominal 3.5 MHz 3.5CL192-3ML convex array transducer was used and connected to a stand alone PC. SASB-THI and DRF-THI scan sequences were recorded interleaved and processed offline. Nineteen patients diagnosed with focal liver pathology were scanned to set a clinical condition, where ultrasonography is often performed. A total of 114 sequences were recorded and evaluated by five radiologists. The evaluators were blinded to the imaging technique, and each sequence was shown twice with different left-right positioning, resulting in 1140 evaluations. The program Image Quality Assessment Program (IQap) and a Visual Analog Scale (VAS) were applied for the evaluation. The scale ranged from -50 to 50 , where positive values favored SASB-THI. SASB-THI and DRF-THI were evaluated alike in $49 \%$ of the evaluations, $28 \%$ favored SASB-THI and $23 \%$ favored DRF-THI. The average rating was 0.70 (Cl: -0.80 to 2.19). The statistical analysis, where the hypothesis of no differences between the techniques was tested, yielded a p-value of $p=0.64$, indicating no preference to any technique. This study demonstrates that SASB-THI and DRF-THI have equally good image quality although a data reduction of 64 times is achieved with SASB-THI.
\end{abstract}

Keywords-Imagine quality evaluation, Synthetic Aperture Sequential Beamforming Tissue Harmonic Imaging, Comparison of techniques

\section{INTRODUCTION}

The default imaging technique in many modern ultrasound scanners is the combination of Dynamic Received Focusing (DRF) and Tissue Harmonic Imaging (THI) [1]. Tissue harmonic imaging (THI) enhances image quality through improvement in contrast, resolution and fewer artifacts in gray scale ultrasonography [2-3]. However, DRF has some drawbacks. The image is acquired using consecutive scan lines, creating constraints on the frame rate. The frame rate is limited by the speed of sound in tissue, the scanning depth, and number of scan lines. Creating a high image quality with a high number of scan lines is thus obtained at the expense of frame rate. Furthermore, the image quality is affected by the fixed transmit with focus at one depth. This is often alleviated by multiple transmit foci. The disadvantage of this is an increased number of emissions, and thereby even further lowering of the frame rate [4].

Another imaging technique, without the same constraints on frame rate, is synthetic aperture (SA). In SA one or a group of active elements emits an unfocused spherical wave, and the scattered signal is received by all the elements. By focusing the data, a low resolution image is obtained, and combining low resolution images from multiple emissions results in a high resolution image with optimal focus in the entire image. The disadvantage of SA is the high computational processing load, which makes implementation of SA on a conventional scanner challenging [5-7]. To solve this problem, synthetic aperture sequential beamforming (SASB) was developed. SASB is a technique that ultilizes a two step beamformer to significantly reduce the computational processing load compared to SA. A set of scan lines are obtained and bearmformed using a fixed focus in both transmit and receive. Then, the image data are beamformed using the fixed focus points as a virtual source to obtain the final synthetic aperture [4,8-9].

SASB use of virtual sources creates an acoustic field intense enough to create harmonics for THI $[1,9]$. To attain the improvement of THI and SASB, this combination was suggested as a new imaging technique $[1,10]$. In a previous preliminary evaluation study with the combination of SASB and THI, SASB-THI attained equally good image quality as DRF-THI [10]. However, this study was only performed with healthy volunteers, so the true clinical relevance could not be clarified. In this study patients with focal liver pathology were scanned in a true clinical setting. This gave the opportunity to study SASB-THI ability to create images on patients. The objective of the study was to determine, if the data reduction achieved by SASB-THI could affect its image quality. The image quality of the SASB-THI was compared to DRF-THI.

\section{MATERIALS AND METHODS}

\section{A. Synthetic Aperture Sequential Beamforming}

In SASB the initial stage beamformer operates on the signals received by the transducer array. The emission sequence scans a focused emission across the volume being imaged. The beamformer applies delay-and-sum beamforming with a fixed delay for each transducer element to create a fixed 
focus scan line. The delay configuration is identical in both transmit and receive. The sample values of a single scan line can be interpreted as the response from a virtual source, emitting a spherical wave, positioned at the focal point of the scan line. The second stage beamformer takes the output of the first stage and uses it as input. The focal point from the first stage beamformer is considered a virtual source, while the samples on the scan line are considered the signal received by a virtual receive element collocated with the virtual source. Each point in the focused image line contains information from a set of spatial positions limited by the opening angle of the virtual source. A single image point is therefore potentially represented in multiple first stage focused scan lines. The second stage beamformer creates a set of image points by combining information from the first stage focused scan lines that contain information from the spatial position of the image point [4,8-9]. Fig. 1 illustrates the system architecture of SASB.
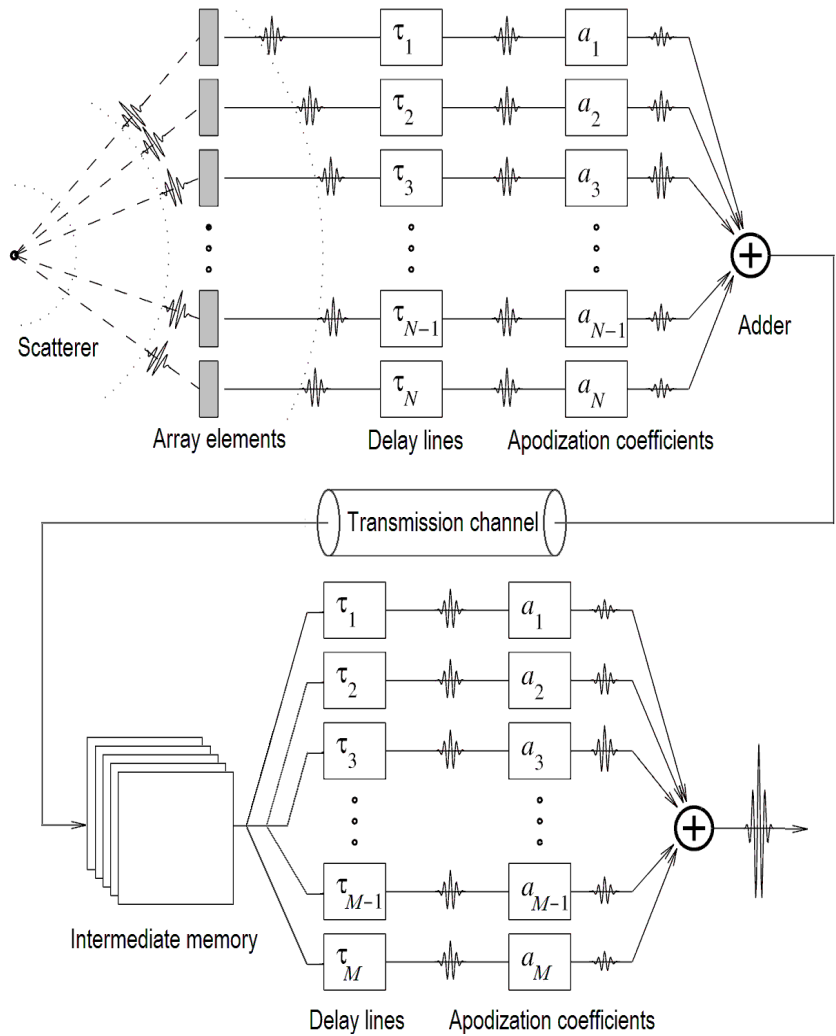

Fig. 1. Illustration of Synthetic Aperture Sequential Bamforming (SASB). For each focused emission a simple receive beamformer create a fixed focused scan line. The scan line is transmitted over a transmission channel and the final image is created in a second dynamic beamformer by refocusing a number of scan lines.

\section{B. Patients}

Nineteen patients (13 males and 6 females) with different focal liver pathologies (liver metastases or hepatocellular carcinoma) were included. The mean age was 65.4 years $(\mathrm{y})$, range: 37-82 y and mean Body Mass Index (BMI) 24.8, range: 16.8-33.0. All patients were admitted at the Department of Surgical Gastroenterology, Copenhagen University Hospital,
Rigshospitalet. Prior to the ultrasound examination pathology was diagnosed with needle biopsy or computed tomography (CT)/magnetic resonance (MR). All patients were scheduled for surgery the day after the ultrasound examination. All patients were included after written informed consent and an approval by the Danish National Committee on Biomedical Research Ethics; journal number H-1-2011-124.

\section{Equipment and data acquisition}

A conventional ultrasound scanner (UltraView 800, BK Medical, Herlev, Denmark) was equipped with a research interface and an abdominal 3.5 MHz 3.5CL192-3ML curved array transducer (Sound Technology Inc., Pennsylvania, USA). A stand alone PC was connected to the ultrasound scanner. Through this setup it was possible to generate image sequences with SASB-THI and DRF-THI interleaved. Images from the same anatomical location were recorded interleaved on a frame basis with both techniques, and sequences ideal for comparison were created (Fig. 2) [11,12]. The frame rate was 8 frames/sec and the scan depth $14.6 \mathrm{~cm}$. Sequences of 3 seconds were recorded. Each patient was scanned in 3 positions where the pathology was clearly visible and in 3 positions where no pathology was visible. All patients were prior to the study scanned with a conventional ultrasound scanner for orientation and if present CT scan and/or MR scan was reviewed to insure correct positioning of the SASB-THI and DRF-THI scan. If the pathology was not visible on the orientation scan the patient was excluded. Six patients were excluded due to this. The medical doctors Andreas Hjelm Brandt and Peter Møller Hansen performed all data collection.

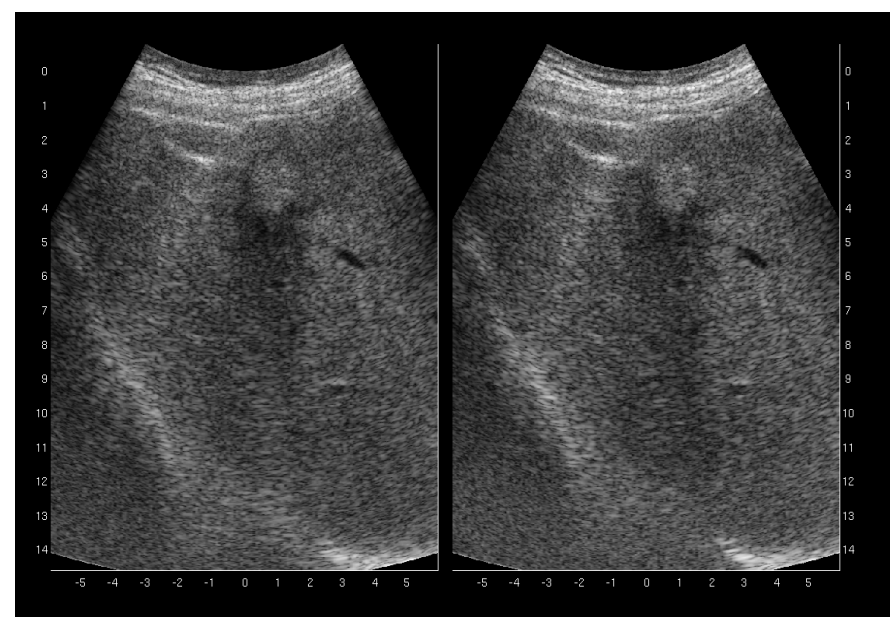

Fig. 2. Example of interleaved recording. SASB-THI is seen on the right side and DRF-THI on the left side.

\section{Image Evalution}

For the image evaluation the in house developed software program Image Quality Assessment Progam (IQap) [12] was applied, and a Visual Analog Scale (VAS) displayed as a sliding bar underneath the sequences [13]. The sliding bar was placed corresponding to which sequence the evaluator rated had the best image quality. If the bar was placed in the middle, the evaluator found no difference between the techniques. The scale ranged from -50 to 50 , where positive values favored 
SASB (Fig. 3). Five radiologists evaluated all sequences. They were blinded to the technique and individually placed in a darkened room during the evaluation. None of them had seen the images beforehand. They were told to make an assessment of image quality regarding contrast, spatial resolution, and unwanted artifacts. During the evaluation each technique was shown side-by-side, so a comparison of the same anatomical location simultaneously was possible with the two techniques. Each sequence was shown twice and randomly switched from left to right, displaying each technique twice.

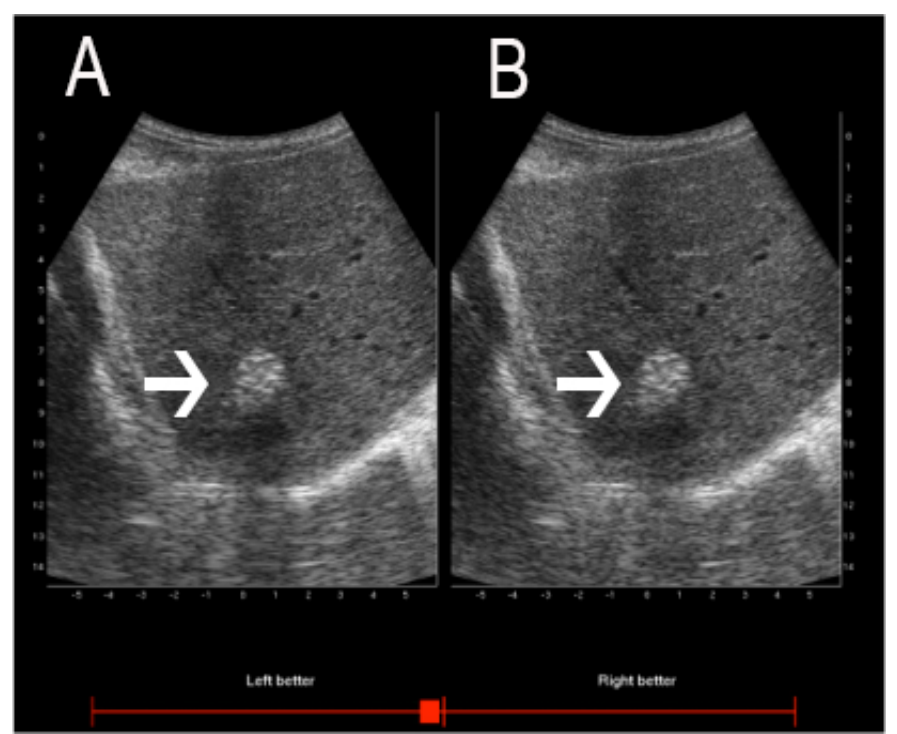

Fig. 3. The figure shows IQap screenshot with the VAS at the bottom. For guidance SASB-THI is here indicated by A, DRF-THI by B and the white arrows are pointing at the focal liver pathology. By dragging the bar to one side the evaluators could specify which technique they preferred.

\section{E. Statistics}

Since each evaluating radiologist most likely has his own interpretation of the VAS in side-by-side image quality comparison, no assumption of normal distribution was made.

The data were analyzed using both a linear mixed model (accounting for dependencies induced by same doctor rating several times and same picture pair showed several times by including these as random effects) and a non-parametric approach based on the Wilcoxon rank sum test, but employing bootstrap techniques to achieve correct $p$-values as the mentioned dependencies yield standard $p$-values invalid. The linear mixed model was used to compute confidence intervals while test of significant differences was based on the nonparametric approach. By also employing a non-parametric approach including bootstrapping it was ensured that the results do not depend on the distributional assumptions of the linear mixed model.

\section{RESULTS}

The evaluation by each radiologist is depicted in Fig. 4. Of all the ratings, no preference was seen to SASB-THI or DRFTHI in $49 \%(555 / 1140)$ of the evaluations, $28 \%(322 / 1140)$ preferred SASB-THI and 23\% (263/1140) preferred DRF-THI. The average rating on the VAS was $0.70(\mathrm{Cl}$ : -0.80 to 2.19$)$.
The statistical analysis, where the hypothesis of no differences between the techniques was tested, yielded a $p$-value of 0.64 .
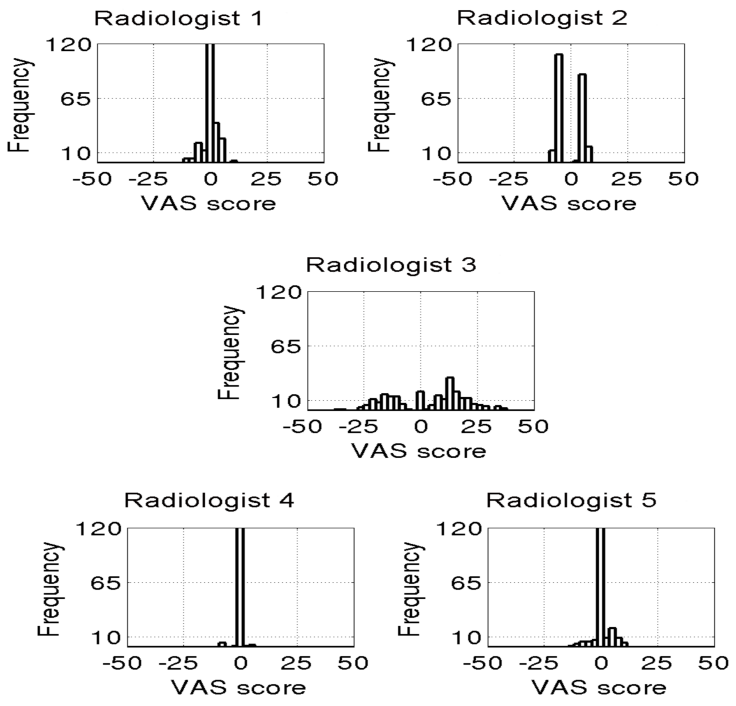

Fig. 4. The figure shows the pooled evaluation of each radiologist.

\section{DISCUSSION}

The combination of SASB and THI has been considered for clinical imaging in this study. Five experienced radiologists rated the image quality of SASB-THI compared to the preferred imaging technique DRF-THI. The results indicate that there is no preference for either technique, thus SASB-THI can generate equally good image quality as the preferred imaging technique. The general comment from the radiologists after the evaluation confirms this result, as they did not notice much of a difference between the various techniques. The predominant advantage of SASB is the reduction in computational processing load compared to SA, but also compared to the DRF. SASB reduces the amount of data transmitted by a factor of 64 compared to DRF. The reduction makes it possible to implement a SA technique in conventional ultrasound scanners, and in the future to construct wireless ultrasound transducers or tablet based ultrasound system [14]. The combination of SASB and THI has the advantage of reduced computational load, and at the same time the benefits of THI e.g. improved contrast and resolution. Is it therefore advisable to further work on implementing SASB-THI in commercial scanners. Compared to the previous preliminary study of SASB-THI, where young slim healthy volunteers were scanned, there were several differences [10]. The clinical patients could not cooperate as well as the healthy volunteers. Due to discomfort lying and pain, some had trouble lying in certain positions and others had trouble holding their breath. In general they were older and had thicker abdominal layers. Some of the patients had previous surgery of the liver, with changed position and appearance of some of the anatomical structures, and with gas-filled intestines moved in areas, so the insonation window of the liver was obscured. Tissue motion 
has in previous in vivo studies with SA been pointed out as a reason for degrading of image quality $[5,15]$. This was reduced in the preliminary study, where the volunteers were asked to lie still and hold their breath while recording the sequences. However, only a few patients were able to comply completely with this [10]. Thus, tissue motion was present on the recordings. It did not affect the image quality evaluation and further confirms SASB-THI ability for clinical imaging. The evaluating radiologist also did not make remarks on tissue motion artifacts. Few clinical studies have so far been performed with SA as imaging technique. The prior studies evaluated breast pathology and the evaluators only had the option of assessing individual still images [16,17]. This study evaluated image sequences, which to our opinion gives a more realistic evaluation of the image quality and more valid assessment of the clinical performance. Although the combination of SASB and THI seems suitable for clinical imaging, there are still several limitations that need to be addressed to replace DRF-THI as the default imaging technique. All sequences were recorded with a relatively low frame rate and in the most "raw" format without any image improving algorithms, e.g. a speckle reduction filter. The images containing pathological changes were therefore not suited for diagnostic evaluation and radiologist were told only to evaluate image quality without considerations on the diagnostic value of the sequences. Further studies with higher frame rate and image quality improving algorithms implemented on SASB-THI could reveal the diagnostic efficacy and usability.

\section{CONCLUSION}

This study is the first study in which SASB-THI has been investigated on patients with pathologic changes. SASB-THI revealed equally good image quality as the preferred imaging technique on a commercial scanner. SASB-THI can be used for clinical imaging and the advantage of reduction in computational processing load can be further exploited to develop wireless transducers.

\section{ACKNOWLEDGMENT}

The authors wish to thank all participating patients at the department of Surgical Gastroenterology, Copenhagen University Hospital, Rigshospitalet and the staff for helping recruiting patients. They also wish to thank M.D. Flemming Jensen, M.D. Caroline Ewertsen, M.D. Trine-Lise Lambine and M.D. Lars Lönn for evaluating the ultrasound recordings. The study was supported by grant number 82-2012-4 from The Danish National Advanced Technology Foundation and by BK Medical.

\section{REFERENCES}

[1] J. Rasmussen, M.C. Hemmsen, S.S. Madsen, P.M. Hansen, M.B. Nielsen, and J.A. Jensen, "Implementation of Tissue Harmonic Synthetic Aperture Imaging on a Commercial Ultrasound System," Proc. IEEE Ultrason. Symp., pp. 121-125, 2012.

[2] R.S. Shapiro, J. Wagreich, R.B. Parsons, A. Stancato-Pasik, H.C. Yeh, and R. Lao, "Tissue harmonic imaging sonography: evaluation of image quality compared with conventional sonography," AJR Am J Roentgenol., vol. 5, pp. 1203-1206, 1998.

[3] H.J. Jang, H.K. Lim, W.J. Lee, S.H. Kim, K.A. Kim, and E.Y. Kim, "Ultrasonographic evaluation of focal hepatic lesions: comparison of pulse inversion harmonic, tissue harmonic, and conventional imaging techniques," J Ultrasound Med, vol. 19 no. 5, pp. 293-299, 2000.

[4] M.C. Hemmsen, P.M. Hansen, T. Lange, J.M. Hansen, K.L. Hansen, M.B. Nielsen, et al, "In vivo evaluation of synthetic aperture sequential beamforming," Ultrasound Med Biol, vol. 38 no.4, pp. 708-716, 2012.

[5] J.A. Jensen, S.I Nikolov, K.L. Gammelmark, and M.H. Pedersen, "Synthetic aperture ultrasound imaging," Ultrasonics, vol. 44 no.1, pp. e5-15, 2006.

[6] I. Trots, A. Nowicki, M. Lewandowski, and Y. Tasinkevych, "Syntetic Aperture Method in Ultrasound Imaging," Archives of Acoustics, vol. 43 no. 4 , pp. 685-695, 2009.

[7] K.-S. Kim and T.-K. Song, "High frame rate and high resolution imaging with synthetic aperture," Key Eng. Mater, vol. 47, no.6, pp. 1510-1519, 2000.

[8] J. Kortbek, J.A. Jensen, and K.L. Gammelmark, "Sequential beamforming for synthetic aperture imaging," Ultrasonics, vol. 53 no.1, pp. 1-16, 2013.

[9] Y. Du, J. Rasmussen, H. Jensen, and J. A. Jensen, "Second harmonic imaging using synthetic aperture sequential beamforming," in Proc. IEEE Ultrason. Symp., pp. 2261-2264, 2011.

[10] J.H. Rasmussen, M.C. Hemmsen, S.S. Madsen, P.M. Hansen, M.B. Nielsen, and J.A. Jensen, "Preliminary study of synthetic aperture tissue harmonic imaging on in-vivo data," Proc. SPIE Medical Imaging, vol. $8675,2013$.

[11] M.C. Hemmsen, S.I Nikolov, M.M. Pedersen, M.J. Pihl, M.S. Enevoldsen, J.M. Hansen et al, "Implementation of a versatile research data acquisition system using a commercially available medical ultrasound scanner," IEEE Trans Ultrason Ferroelectr Freq Control, vol. 59 no. 7, pp. 1487-1499, 2012.

[12] M.C. Hemmsen, M.M. Pedersen, S.I. Nikolov, M.B. Nielsen, J.A. Jensen, "Ultrasound image quality assessment: A framework for evaluation of clinical image quality," Proc. SPIE Medical Imaging, vol. 7629, 2010.

[13] M. Freyd, "The Graphic Rating Scale,” J Educ Psychol, vol. 14 no.2, pp 83-102, 1923

[14] P.M. Hansen, M.C. Hemmsen, A. H. Brandt, J. Rasmussen, T. Lange, P.S. Krohn, L. Lönn, J.A. Jensen, and M.B. Nielsen, "Clinical Evaluation of Synthetic Aperture Sequential Beamforming Ultrasound in Patients with Liver Tumors" accepted for publication, july 2014.

[15] M.H. Pedersen, K.L. Gammelmark, and J.A. Jensen, "In-Vivo Evaluation of Convex Array Synthetic Aperture Imaging," Ultrasound Med Biol, vol. 33 no.1, pp. 37-47, 2007.

[16] C. Kim, C.Yoon, J.H. Park, Y. Lee, W.H Kim, J.M Chang, B.I. Choi, T.K. Song, and Y.M. Yoo, "Evaluation of Ultrasound Synthetic Aperture Imaging Using Bidirectional Pixel-Based Focusing: Preliminary Phantom and In Vivo Breast Study," Proc. IEEE Trans Biomed Eng., vol.60 no.10, pp. 2716-2724, 2013.

[17] W.H. Kim, J.M. Chang, C. Kim, J. Park, Y. Yoo, W.K. Moon, N. Cho, and B.I. Choi, "Synthetic Aperture Imaging in Breast Ultrasound: A Preliminary Clinical Study," Acad Radiol, vol. 19 no.8, pp.923-929, 2012 . 